\title{
PERIODS OF STREBEL DIFFERENTIALS AND ALGEBRAIC CURVES DEFINED OVER THE FIELD OF ALGEBRAIC NUMBERS *
}

\author{
MOTOHICO MULASE ${ }^{\dagger}$ AND MICHAEL PENKAVA ${ }^{\ddagger}$
}

\begin{abstract}
In [8] we have shown that if a compact Riemann surface admits a Strebel differential with rational periods, then the Riemann surface is the complex model of an algebraic curve defined over the field of algebraic numbers. We will show in this article that even if all geometric data are defined over $\overline{\mathbb{Q}}$, the Strebel differential can still have a transcendental period. We construct a Strebel differential $q$ on an arbitrary complete nonsingular algebraic curve $C$ defined over $\overline{\mathbb{Q}}$ such that (i) all poles of $q$ are $\overline{\mathbb{Q}}$-rational points of $C$; (ii) the residue of $\sqrt{q}$ at each pole is a positive integer; and (iii) $q$ has a transcendental period.
\end{abstract}

1. Introduction. The periodic function $e^{i \pi \theta}$ has the following remarkable property: for every rational number $\theta, e^{i \pi \theta}$ gives an algebraic number, while for every non-rational algebraic number $\theta$, it gives a transcendental number. It follows that if $e^{i \pi \theta}$ is algebraic and $\theta$ is not rational, then $\theta$ is transcendental. This somewhat reciprocal algebraicity-transcendence relation between the periods of a function and its values is commonly seen among the periodic functions appearing in algebraic geometry such as modular functions and theta functions [1].

The purpose of this paper is to examine a similar relation between the periods of Strebel differentials on a complete nonsingular algebraic curve and its field of definition. In an earlier paper [8], we have established that if a compact Riemann surface $C$ admits a Strebel differential $q$ such that the length of every critical horizontal trajectory is rational, then $C$ is the complex model of an algebraic curve defined over the field $\overline{\mathbb{Q}}$ of algebraic numbers. (We refer to [8] for the definitions of the notions used in this article.) Let us call the length of a critical horizontal trajectory a period of $q$. Thus the rationality of the periods makes the field of definition of $C$ algebraic. Following the analogy of the relation between the periods and the values of theta functions, one can ask questions about the transcendence of the periods of a Strebel differential and the field of definition of an algebraic curve.

Let $\Gamma$ be a ribbon graph, that is, a graph with a prescribed cyclic order of halfedges at each vertex. A ribbon graph is a metric ribbon graph if a positive real number, conventionally referred to as the length, is assigned to each edge. From every metric ribbon graph $\Gamma$, one can construct a compact Riemann surface $C$ and a Strebel differential $q$ on it in a unique manner. The number of poles of $q$ is equal to the number of boundary circuits of $\Gamma$, and the residue of $\sqrt{q}$ at each pole is the total length of the boundary circuit corresponding to the pole.

Conversely, starting with a compact Riemann surface $C$ with $n>0$ marked points and an $n$-tuple of positive real numbers, one constructs a unique metric ribbon graph as the union of the critical horizontal trajectories of the Strebel differential $q$ on $C$. The integral of $\sqrt{q}$ between zeros of $q$ along a critical horizontal trajectory is called a period of $q$. The periods of the Strebel differential determine a metric on the ribbon graph.

${ }^{*}$ Received July 17, 2001; accepted for publication November 12, 2002.

†Department of Mathematics, University of California, Davis, CA 95616-8633, USA (mulase@math.ucdavis.edu). Research supported by NSF Grant DMS-9971371 and the University of California, Davis.

$\ddagger$ Department of Mathematics, University of Wisconsin, Eau Claire, WI 54702-4004, USA (penkavmr@uwec.edu). Research supported in part by NSF Grant DMS-9971371 and the University of Wisconsin-Eau Claire. 
Harer [4] used this idea to establish an orbifold isomorphism

$$
\coprod_{\Gamma} \frac{\mathbb{R}_{+}^{e(\Gamma)}}{\operatorname{Aut}_{\partial}(\Gamma)} \stackrel{\sim}{\longrightarrow} \mathfrak{M}_{g, n} \times \mathbb{R}_{+}^{n}
$$

where $\Gamma$ runs over all ribbon graphs with valence of each vertex no less than 3 and with Euler characteristic $2-2 g-n, e(\Gamma)$ is the number of edges of $\Gamma, \operatorname{Aut}_{\partial}(\Gamma)$ is the group of ribbon graph automorphisms of $\Gamma$ that fix its boundary circuits, and $\mathfrak{M}_{g, n}$ is the moduli space of smooth compact complex algebraic curves of genus $g$ with $n$ ordered marked points. This isomorphism plays a key role in many recent papers (cf. [3], [5], [6], [7], [9], [12]). For an explicit construction of this isomorphism, we refer to $[8]$.

The set of rational points

$$
\coprod_{\Gamma} \frac{\mathbb{Q}_{+}^{e(\Gamma)}}{\operatorname{Aut}_{\partial}(\Gamma)} \subset \coprod_{\Gamma} \frac{\mathbb{R}_{+}^{e(\Gamma)}}{\operatorname{Aut}_{\partial}(\Gamma)}
$$

is well defined (although not as an orbifold over $\mathbb{Q}$ ) because a ribbon graph automorphism acts as a permutation of edges. Let $\mathfrak{M}_{g, n}(\overline{\mathbb{Q}})$ denote the moduli space of $n$-pointed complete nonsingular algebraic curves defined over $\overline{\mathbb{Q}}$. Comparing Belyi's theorem [2], Grothendieck's idea of dessins d'enfants [10], and Strebel theory [11], we have established in [8] that there is a natural injective map $j$ :

$$
\begin{array}{ccc}
\coprod_{\Gamma} \frac{\mathbb{R}_{+}^{e(\Gamma)}}{\operatorname{Aut}_{\partial}(\Gamma)} \stackrel{\sim}{\longrightarrow} \mathfrak{M}_{g, n} \times \mathbb{R}_{+}^{n} \\
\bigcup & \cup \\
\coprod_{\Gamma} \frac{\mathbb{Q}_{+}^{e(\Gamma)}}{\operatorname{Aut}_{\partial}(\Gamma)} \underset{j}{\longrightarrow} \mathfrak{M}_{g, n}(\overline{\mathbb{Q}}) \times \mathbb{Q}_{+}^{n} .
\end{array}
$$

Belyi's theorem shows that every complete nonsingular algebraic curve over $\overline{\mathbb{Q}}$ is constructed as the image of the map $j$ if we do not specify the number $n$ of marked points. However, in the light of the geometric $G a l(\overline{\mathbb{Q}} / \mathbb{Q})$ actions $[10], j$ cannot be surjective. Indeed, we shall prove the following:

THEOREM 1. Let $C$ be an arbitrary complete nonsingular algebraic curve defined over $\overline{\mathbb{Q}}$. Then there is a Strebel differential $q$ on the complex model of $C$ such that

1. every pole of $q$ is a $\overline{\mathbb{Q}}$-rational point of $C$;

2. the residue of $\sqrt{q}$ at each pole is a positive integer; and

3. $q$ has a transcendental period.

Acknowledgement: The authors would like to thank Josephine Yu for creating the graphics Figure 1 and Figure 2 in this paper.

2. Construction of the Strebel Differential. Let us start by constructing a simple example on $\mathbb{P}^{1}$. We wish to exhibit a Strebel differential $q_{c}$ on $\mathbb{P}^{1}$ that has poles at $0,1, \infty, c$ and $c^{2}$, such that $\sqrt{q_{c}}$ has residues $2,2,2,4$, and 2 , resp., at these poles, with $c \in \overline{\mathbb{Q}} \backslash\{0,1\}$ a constant to be determined later. We define two rational maps $f$ and $g$ in order to construct certain Strebel differentials on $\mathbb{P}^{1}$. First we choose

$$
f: \mathbb{P}^{1} \ni x \longmapsto y=\frac{1}{(1-c)^{2}} \frac{(x-c)^{2}}{x} \in \mathbb{P}^{1} .
$$


Since

$$
f^{\prime}(x)=\frac{1}{(1-c)^{2}} \frac{x^{2}-c^{2}}{x^{2}},
$$

$f$ is ramified at $x= \pm c$. We note that

$$
f(0)=f(\infty)=\infty, \quad f(1)=f\left(c^{2}\right)=1, \quad f(c)=0, \quad f(-c)=-\frac{4 c}{(1-c)^{2}}
$$

The other rational map is

$$
g: \mathbb{P}^{1} \ni y \longmapsto \zeta=\frac{4\left(y^{2}-y+1\right)^{3}}{27 y^{2}(1-y)^{2}} \in \mathbb{P}^{1} .
$$

Consider the meromorphic quadratic differential

$$
q_{0}=\frac{1}{4 \pi^{2}} \frac{d \zeta^{2}}{\zeta(1-\zeta)}
$$

on $\mathbb{P}^{1}$, and put

$$
q_{1}=g^{*}\left(q_{0}\right)=-\frac{1}{\pi^{2}} \frac{y^{2}-y+1}{y^{2}(1-y)^{2}} d y^{2}
$$

It has quadratic poles at 0,1 , and $\infty$, and simple zeros at $\frac{1}{2} \pm i \frac{\sqrt{3}}{2}$. The residue of $\sqrt{q_{1}}$ at each pole is 2 , and the three periods of $q_{1}$ are all 1 . Let $\Gamma_{y}=g^{-1}([0,1])$. It has been shown in [8] that $\Gamma_{y}$ is the ribbon graph consisting of the set of critical trajectories of the Strebel differential $q_{1}$.

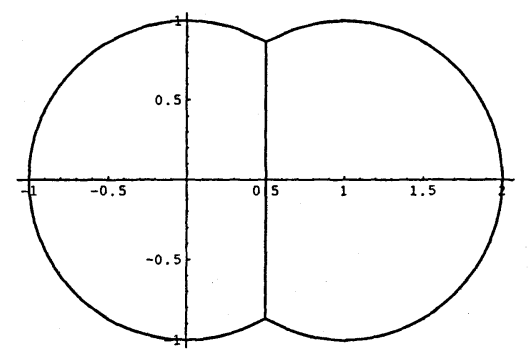

FIG. 1. Graph $\Gamma_{y}$, the inverse image of $[0,1]$ via $\zeta=\frac{4\left(y^{2}-y+1\right)^{3}}{27 y^{2}(1-y)^{2}}$.

Next, let $s$ be a real algebraic number such that $0<s<\frac{\sqrt{3}}{2}$, choose the constant $c$ so that

$$
f(-c)=-\frac{4 c}{(1-c)^{2}}=\frac{1}{2}+i s
$$

and define $q_{c}=f^{*}\left(q_{1}\right)$. Since the two critical values of the double-sheeted holomorphic covering map $f$ are $f(c)=0$ and $f(-c)$, one sees that the Strebel differential $q_{c}$ on $\mathbb{P}^{1}$ has quadratic poles at $0,1, \infty, c$ and $c^{2}$ with residues of $\sqrt{q_{c}}$ at these poles 2,2 , 2,4 , and 2 , resp., and a new double zero at $-c$. Let $\Gamma_{c}=f^{-1}\left(\Gamma_{y}\right)$. Because of the choice of $c$ in Eq. 6, $\Gamma_{c}$ is the ribbon graph consisting of the critical trajectories for 


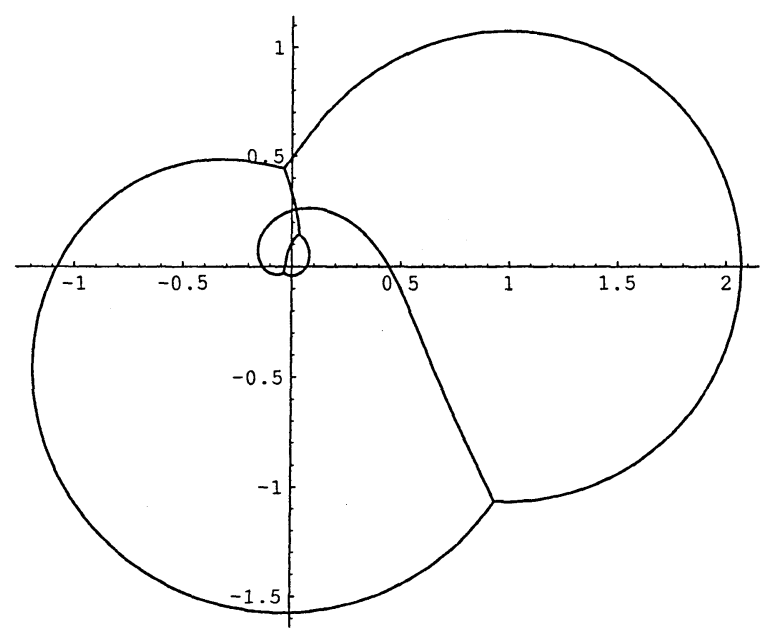

FIG. 2. Graph $\Gamma_{c}$ for $s=\frac{5 \sqrt{3}}{11}$.

the Strebel differential $q_{c}$. It has four tri-valent vertices at $f^{-1}\left(\frac{1}{2} \pm i \frac{\sqrt{3}}{2}\right)$ and a unique 4-valent vertex at $-c=f^{-1}\left(\frac{1}{2}+i s\right)$.

Let $L$ denote the edge of $\Gamma_{c}$ that connects the vertex $-c$ and one of the tri-valent vertices. From Eq. 5 one sees that the period of $q_{c}$ corresponding to the edge $L$ is equal to $\frac{1}{2} \pm \ell(s)$ depending on the choice of the tri-valent vertex, where

$$
\begin{aligned}
\ell(s) & =\int_{1 / 2}^{1 / 2+i s} \sqrt{-\frac{1}{\pi^{2}} \frac{y^{2}-y+1}{y^{2}(1-y)^{2}}} d y \\
& =\frac{1}{\pi} \int_{0}^{s} \frac{\sqrt{3 / 4-t^{2}}}{1 / 4+t^{2}} d t \\
& =\frac{1}{\pi} \arcsin \left(\frac{2 s\left(9+4 s^{2}\right)}{3 \sqrt{3}\left(1+4 s^{2}\right)}\right) .
\end{aligned}
$$

In Section 3, we shall show that $\ell(s)$ is transcendent at $s=r \sqrt{3}$ for every rational number $r$ such that $0<r<\frac{1}{2}$.

The above construction immediately gives the construction of the Strebel differential $q$ for the general case of Theorem 1 . Let $C$ be an arbitrary complete nonsingular algebraic curve defined over $\overline{\mathbb{Q}}$. Belyi [2] has shown that there is a holomorphic map

$$
\beta: C \longrightarrow \mathbb{P}^{1}
$$

that is ramified only over 0,1 and $\infty$. The map $\beta$ is called a Belyi map. Without loss of generality, we can assume that the ramification degrees over the point $0 \in \mathbb{P}^{1}$ are no less than 3 and the ramification degrees over $1 \in \mathbb{P}^{1}$ are always 2. Define $q=\beta^{*}\left(q_{c}\right)$ and $\Gamma=\beta^{-1}\left(\Gamma_{c}\right)$. Then $q$ is the Strebel differential on $C$ with poles at $\beta^{-1}(0), \beta^{-1}(1)$, $\beta^{-1}(\infty), \beta^{-1}(c)$, and $\beta^{-1}\left(c^{2}\right)$. The residue of $\sqrt{q}$ at each of these poles is 2 times the ramification degree of $\beta$ at each ramification point, 4 at each inverse image of $c$, and 2 at each inverse image of $c^{2}$. Certainly the period of $q$ corresponding to any edge of $\Gamma$ that is an inverse image of $L$ is a transcendental number if we chose $s=r \sqrt{3}$.

3. Transcendence of the Period. Let us now show that $\ell(s)$ of Eq. 7 is transcendental for every $s=r \sqrt{3}$, where $r$ is a rational number in between 0 and $1 / 2$. 
Since

$$
\sin (\pi \ell(s))=\frac{2 s\left(9+4 s^{2}\right)}{3 \sqrt{3}\left(1+4 s^{2}\right)}=\frac{2 r\left(3+4 r^{2}\right)}{\left(1+12 r^{2}\right)} \in \mathbb{Q} \backslash\{1 / 2\},
$$

the claim follows from

Proposition 2. Let $0<\ell<1 / 2$ be such that $a=\sin (\pi \ell)$ is rational but not equal to $\frac{1}{2}$. Then $\ell$ is transcendental.

Proof. Let

$$
b=-a+i \sqrt{1-a^{2}}=e^{i \pi(\ell+1 / 2)}
$$

This is a solution of the quadratic equation $x^{2}+2 a x+1=0$. Let $a=m / n$ be an irreducible fraction. Then $n x^{2}+2 m x+n$ is primitive and irreducible if $n$ is odd, and if $n=2 k$, then $k x^{2}+m x+k$ is primitive and irreducible.

Suppose that $\ell \in \mathbb{Q}$. Then $b$ is a solution of the primitive equation $x^{N}-1=0$ for some integer $N$. We note that the primitive minimal polynomial of $b$ divides $x^{N}-1$. Therefore, if $n$ is odd, then $n=1$, and hence $a=m \geq 1$, which is a contradiction. If $n$ is even, then $k=1$ and the only possibility is $a=\frac{1}{2}$. Thus $\ell$ is not rational. It cannot be algebraic because $b$ is algebraic. This completes the proof.

It would be desirable to establish that if a compact Riemann surface $C$ admits a Strebel differential $q$ whose periods are algebraic but not rational, then the geometric data $\left(C,\left(p_{1}, \cdots, p_{n}\right)\right)$, where $\left(p_{1}, \cdots, p_{n}\right)$ are the poles of $q$, cannot be defined over $\overline{\mathbb{Q}}$. In our simple example of Section 2 , if the period corresponding to the edge $L$ is taken to be algebraic but not rational, then the ramification points $c$ and $c^{2}$ become transcendental. However, we do not have any general theorem in this direction.

\section{REFERENCES}

[1] Alan Baker, Transcendental number theory, Cambridge University Press, 1990.

[2] G. V. BELYI, On galois extensions of a maximal cyclotomic fields, Math. U.S.S.R. Izvestija, 14 (1980), pp. 247-256.

[3] D. Bessis, C. ITZYKSON AND J. B. ZuBER, Quantum field theory techniques in graphical enumeration, Advanced in Applied Mathematics, 1 (1980), pp. 109-157.

[4] JOHN L. HARER, The virtual cohomological dimension of the mapping class group of an orientable surface, Inventiones Mathematicae, 84 (1986), pp. 157-176.

[5] John L. HARER AND DON ZAGIER, The Euler characteristic of the moduli space of curves, Inventiones Mathematicae, 85 (1986), pp. 457-485.

[6] Maxim KonTsevich, Intersection Theory on the Moduli Space of Curves and the Matrix Airy Function, Communications in Mathematical Physics, 147 (1992), pp. 1-23.

[7] Мотонico Mulase, Asymptotic analysis of a hermitian matrix integral, International Journal of Mathematics, 6 (1995), pp. 881-892.

[8] Motohico Mulase and Michael PenkaVa, Ribbon graphs, quadratic differentials on Riemann surfaces, and algebraic curves defined over $\overline{\mathbb{Q}}$, Asian Journal of Mathematics, 2 (1998), pp. 875-920.

[9] Robert C. Penner, Perturbation series and the moduli space of Riemann surfaces, Journal of Differential Geometry, 27 (1988), pp. 35-53.

[10] LeIlla SChNePS AND PIERRE LOCHAK, editors, Geometric Galois actions: Around Grothendieck's esquisse d'un programme, London Mathematical Society Lecture Notes Series, vol. 242, 1997.

[11] Kurt Strebel, Quadratic differentials, Springer-Verlag, 1984.

[12] EDWARD WITTEN, Two dimensional gravity and intersection theory on moduli space, Surveys in Differential Geometry, 1 (1991), pp. 243-310. 
